\title{
CARACTERÍSTICAS FISICAS E QUÍMICAS DO LÁTEX E CRESCIMENTO DA SERINGUEIRA EM FUNÇÃO DA CALAGEM E DA ADUBAÇÃO NPK EM DOIS SISTEMAS DE EXPLOTAÇÃO
}

\author{
ADONIAS DE CASTRO VIRGENS FILHO ${ }^{1}$ \\ ADÔNIS MOREIRA ${ }^{2}$ \\ PAULO ROBERTO DE CAMARGO E CASTRO ${ }^{3}$
}

\begin{abstract}
RESUMO - Objetivando avaliar os efeitos de diferentes combinações de NPK, com e sem calagem, sobre as características físicas e químicas do látex e as condições de explotação da seringueira (Hevea sp.), clone RRIM 600, foi conduzido um experimento em blocos casualizados com parcelas subdivididas contendo oito tratamentos: sem adubação e sem calagem (T1), sem adubação e com calagem (T2), $\mathrm{N}_{1} \mathrm{P}_{1} \mathrm{~K}_{0}+$ calagem (T3), $\mathrm{N}_{2} \mathrm{P}_{2} \mathrm{~K}_{0}+$ calagem (T4), $\mathrm{N}_{1} \mathrm{P}_{1} \mathrm{~K}_{1}+$ calagem (T5), $\mathrm{N}_{2} \mathrm{P}_{2} \mathrm{~K}_{1}+$ calagem (T6), $\mathrm{N}_{1} \mathrm{P}_{1} \mathrm{~K}_{2}+$ calagem (T7) e $\mathrm{N}_{2} \mathrm{P}_{2} \mathrm{~K}_{2}+$ calagem (T8). Nas duas subparcelas, foram utilizados os seguintes sistemas de explotação: (S1) ${ }^{1 / S} \mathrm{~d} / 46 \mathrm{~d} / 7$ ET 2,5\% LaPa 1/1 10/y e (S2) 1/S d/6 6 d/7 ET 5,0\% LaPa 1/1 10/y com quatro repetições. As
\end{abstract}

doses utilizadas corresponderam a 0,40 e $80 \mathrm{~kg} \mathrm{ha}^{-1}$ ano $^{-1}$ de $\mathrm{N}, 0,17,5$ e $35,0 \mathrm{~kg} \mathrm{ha}^{-1}$ ano $^{-1}$ de $\mathrm{P}_{2} \mathrm{O}_{5}$ e 0 , 33,2 e $66,4 \mathrm{~kg} \mathrm{ha}^{-1}$ ano $^{-1}$ de $\mathrm{K}_{2} \mathrm{O}$. Pelos resultados, verificou-se que o incremento no perímetro do tronco não foi influenciado pelo efeito da interação entre 'adubação+calagem' vs. 'sistemas de explotação'. O aumento da espessura da casca foi afetado pelos tratamentos ' $\mathrm{N}_{0} \mathrm{P}_{0} \mathrm{~K}_{0}+$ calagem' e 'calagem+adubação', quando comparado com o tratamento $\mathrm{N}_{0} \mathrm{P}_{0} \mathrm{~K}_{0}$ sem calagem'. Plantas submetidas ao tratamento $\mathrm{N}_{1} \mathrm{P}_{1} \mathrm{~K}_{0}$ + calagem e ao sistema $1 / 8 \mathrm{~d} / 66 \mathrm{~d} / 7$ ET 5\% 10/y apresentam maiores concentrações de sacarose no látex.

TERMOS PARA INDEXAÇÃO: Hevea brasiliensis, perímetro do troco, espessura da casca, RRIM 600, diagnose do látex.

\section{LATEX PHYSICAL AND CHEMISTRY CHARACTERISTICS AND GROWTH OF RUBBER TREE AS A FUNCTION OF LIME AND NPK FERTILIZATION IN TWO TAPPING SYSTEMS}

\begin{abstract}
The objective of this study was to evaluated physiological latex characteristics and the tapping systems of the clone RRIM 600 of rubber tree (Hevea sp.) an experiment was conducted with continuous supply NPK fertilizers with or without lime. The experiment was conducted in a randomized splitplot design with eight treatments: without fertilizer and without lime (T1), without fertilizer and with lime (T2), $\mathrm{N}_{1} \mathrm{P}_{1} \mathrm{~K}_{0}+$ lime (T3), $\mathrm{N}_{2} \mathrm{P}_{2} \mathrm{~K}_{0}+$ lime (T4), $\mathrm{N}_{1} \mathrm{P}_{1} \mathrm{~K}_{1}$ + lime (T5), $\mathrm{N}_{2} \mathrm{P}_{2} \mathrm{~K}_{1}+$ lime (T6), $\mathrm{N}_{1} \mathrm{P}_{1} \mathrm{~K}_{2}+$ lime (T7) e $\mathrm{N}_{2} \mathrm{P}_{2} \mathrm{~K}_{2}+$ lime (T8). In the two sub-treatments the
\end{abstract}

following tapping systems were used: (S1) $1 / 8 \mathrm{~d} / 46 \mathrm{~d} / 7$ ET $2.5 \%$ LaPa 1/1 10/y e (S2) $1 / 8 \mathrm{~d} / 66 \mathrm{~d} / 7$ ET $5.0 \%$ $\mathrm{LaPa} 1 / 110 / \mathrm{y}$ with four replicates. The rates used were 0,40 and $80 \mathrm{~kg} \mathrm{ha}^{-1} \mathrm{y}^{-1} ; 0,17.5$ e $35.0 \mathrm{~kg} \mathrm{ha}^{-1} \mathrm{y}^{-1} \mathrm{de}$ $\mathrm{P}_{2} \mathrm{O}_{5}$ e $0,33.2$ e $66.4 \mathrm{~kg} \mathrm{ha}^{-1} \mathrm{y}^{-1}$ de $\mathrm{K}_{2} \mathrm{O}$. The interaction of fertilizer with or without lime versus tapping system did not affect trunk girth increment. The bark thickness was significantly increased with liming, and $\mathrm{N}_{1} \mathrm{P}_{1} \mathrm{~K}_{0}$ and tapping system $1 / 8 \mathrm{~d} / 66 \mathrm{~d} / 7$ ET $5 \%$ 10/y increased latex saccharose concentration.

INDEX TERMS: Hevea brasiliensis, RRIM 600, trunk growth increment, bark of thickness, latex diagnosis.

1. Engenheiro Agrônomo, Dr., CEPLAC/CEPEC, Caixa Postal 07, 45.600-000 - Itabuna, BA. adoniascastro@uol.com.br

2. Engenheiro Agrônomo, Dr., Embrapa Amazônia Ocidental (CPAA), Caixa Postal 319, 69.011-970 - Manaus, AM. Bolsista CNPq. adonis@cpaa.embrapa.br

3. Engenheiro Agrônomo, Dr., Depto de Ciências Biológicas - ESALQ/USP, Caixa Postal 96, 13.418-900 Piracicaba, SP. pcrcastr@carpa.ciagri.usp.br 


\section{INTRODUÇÃO}

Em um seringal, as raízes exploram os nutrientes do solo, utilizando-os na formação de fitomassa e grande parte retorna ao solo com a queda das folhas e ramos, formando uma densa camada de serrapilheira que, posteriormente, se decompõe. Nesse ecossistema, as perdas de nutrientes ocorrem em razão da extração do látex, retirada da madeira, lixiviação e erosão do solo. A apreciação da dinâmica desse ecossistema é fundamental para o entendimento dos requerimentos nutricionais da cultura (WATSON, 1989).

Mainstone (1963), estudando a adubação mineral da seringueira, observou uma rápida resposta no teor de nutrientes nas folhas, enquanto o seu efeito na produção foi demorado. Pushparajah (1969) obteve resposta positiva na produção com aplicação de potássio em seringal adulto na Malásia, sobretudo quando fez aplicação de estimulantes de fluxo do látex. Os incrementos da produção de borracha seca foram elevados, e esses resultados foram inversos aos obtidos por Reis e Cabala Rosand (1988), nas condições do sudoeste da Bahia.

Vários métodos têm sido utilizados para monitorar as condições fisiológicas das plantas na fase de sangria. Ferrand (1944) mostra uma relação direta entre o perímetro do tronco, a espessura da casca da seringueira e a produção de borracha seca. Para Jacob et al. (1989) e Kekwick (1989), o método mais preciso é o diagnóstico do látex. Isso é possível pela associação de informações de campo com características fisiológicas relacionadas ao metabolismo da síntese do isopreno, que é a molécula da borracha da seringueira. Por meio dessa técnica, torna-se possível adequar a intensidade de explotação dos seringais, levando-se em conta as características clonais e a influência dos fatores ambientais, procurando-se, assim, otimizar a produção, mantendo as plantas em condições de equilíbrio fisiológico (JACOB et al., 1995).

A análise do látex pelas características fisiológicas possibilita a definição de uma condição de sub ou superexplotação. Tal conclusão pode ser obtida pela avaliação da influência de cada variável analisada no látex e pela correlação entre essas e a duração do fluxo de látex e a regeneração de isopreno 'in situ' no sistema laticífero, entre duas sangrias. Para tanto, são definidos valores para extrato seco, sacarose, tióis, fósforo inorgânico, magnésio e pH (JACOB et al., 1987; JACOB et al., 1988).

Objetivou-se com este estudo avaliar o efeito da calagem e da adubação NPK sobre o crescimento e as características físicas e químicas do látex de seringueiras, com 16 anos de campo, cultivadas no Planalto Ocidental Paulista

\section{MATERIAL E MÉTODOS}

O início do experimento ocorreu em fevereiro de 1995, quando as plantas apresentavam 16 anos de idade e um perímetro médio de $0,60 \mathrm{~m}$ à altura de $1,50 \mathrm{~m}$ do solo (DAP), sendo esse conduzido durante três anos na Fazenda São José do Seringal Paulista, localizada no município de Buritama, Estado de São Paulo.

O clima da região é quente, de inverno seco, caracterizado como Cwa de acordo com a classificação de Köeppen (SETZER, 1946). A precipitação pluviométrica média é de $1200 \mathrm{~mm}$, com média mensal mínima de $30 \mathrm{~mm}$ no inverno, umidade relativa média de $68 \%$ e temperatura média anual de $22^{\circ} \mathrm{C}$ (NASCIMENTO e PEREIRA, 1988). Essa região é caracterizada como preferencial para a heveicultura, com condições térmica e hídrica satisfatórias (ORTOLANI et al., 1983). O solo é um Argissolo com boa drenagem, apresentando as seguintes características químicas na profundidade de 0 $20 \mathrm{~cm}$, quando o experimento foi implantado: $\mathrm{pH}$ em $\mathrm{CaCl}_{2}=3,8$; M.O. $=12,4 \mathrm{~g} \mathrm{dm}^{-3} ; \mathrm{P}_{\text {(resina) }}=1,1 \mathrm{mg} \mathrm{dm}^{-3}$; $\mathrm{K}=0,8 \mathrm{mmol}_{\mathrm{c}} \mathrm{dm}^{-3} ; \mathrm{Ca}=2,0 \mathrm{mmol}_{\mathrm{c}} \mathrm{dm}^{-3} ; \mathrm{Mg}=1,9$ $\mathrm{mmol}_{\mathrm{c}} \mathrm{dm}^{-3} ; \mathrm{Al}^{3+}=10,8 \mathrm{mmol}_{\mathrm{c}} \mathrm{dm}^{-3} ; \mathrm{H}^{+}+\mathrm{Al}^{3+}=29,8$ $\mathrm{mmol}_{\mathrm{c}} \mathrm{dm}^{-3} ; \mathrm{SB}=4,8 \mathrm{mmol}_{\mathrm{c}} \mathrm{dm}^{-3} ; \mathrm{V}=13,7 \%$, argila $=$ $177 \mathrm{~g} \mathrm{~kg}^{-1}$, limo $=75 \mathrm{~g} \mathrm{~kg}^{-1}$ e areia $=748 \mathrm{~g} \mathrm{~kg}^{-1}$.

O material botânico utilizado foi o clone RRIM 600 , enxertado sobre mudas provenientes de sementes ilegítimas de Hevea brasiliensis M. Arg. Na condução do experimento, foram feitos o controle preventivo das doenças do painel (FURTADO e SILVEIRA, 1990) e a quebra de galhos caídos nas entrelinhas, a fim de facilitar a sua incorporação no solo. No controle das plantas invasoras, fezse uma roçagem mecânica por ano, sendo pequena a ocorrência de plantas daninhas no sub-bosque do seringal.

$\mathrm{O}$ delineamento experimental foi em blocos casualizados com parcelas subdivididas com oito tratamentos principais, dois tratamentos secundários, quatro repetições, 24 plantas úteis por parcela e 12 por subparcela. Os tratamentos principais (adubação) envolveram duas testemunhas, sendo uma sem adubação e sem calagem (T1) e outra sem adubação e com calagem (T2), além de outros seis com adubação e com calagem, sendo eles: $\mathrm{N}_{1} \mathrm{P}_{1} \mathrm{~K}_{0}$ (T3), $\mathrm{N}_{2} \mathrm{P}_{2} \mathrm{~K}_{0}$ (T4), $\mathrm{N}_{1} \mathrm{P}_{1} \mathrm{~K}_{1}$ (T5), $\mathrm{N}_{2} \mathrm{P}_{2} \mathrm{~K}_{1}$ (T6), $\mathrm{N}_{1} \mathrm{P}_{1} \mathrm{~K}_{2}$ (T7) e $\mathrm{N}_{2} \mathrm{P}_{2} \mathrm{~K}_{2}$ (T8). As quantidades, em kg ha ${ }^{-1}$ ano $^{-1}$, de $\mathrm{N}, \mathrm{P}_{2} \mathrm{O}_{5}$ e $\mathrm{K}_{2} \mathrm{O}$ foram: $\mathrm{N}_{0}=0$; $\mathrm{N}_{1}=40 ; \mathrm{N}_{2}=80 ; \mathrm{P}_{0}=0$ de $\mathrm{P}_{2} \mathrm{O}_{5} ; \mathrm{P}_{1}=17,5$ de $\mathrm{P}_{2} \mathrm{O}_{5} ; \mathrm{P}_{2}$ $=35,0$ de $\mathrm{P}_{2} \mathrm{O}_{5} ; \mathrm{K}_{\mathrm{o}}=0$ de $\mathrm{K}_{2} \mathrm{O} ; \mathrm{K}_{1}=33,2$ de $\mathrm{K}_{2} \mathrm{O}$ e $\mathrm{K}_{2}$ 
$=66,4$ de $\mathrm{K}_{2} \mathrm{O}$. Com relação à calagem, adotou-se como critério a elevação da saturação por bases a $50 \%$ (CARDOSO, 1992). O calcário (24\% de CaO, $16 \%$ de $\mathrm{MgO}$ e PRNT de 61\%) foi distribuído a lanço e em cobertura na área total.

A adubação fosfatada (superfosfato simples) foi realizada em uma só aplicação no decorrer do período chuvoso de 1995, e no início das chuvas nos anos de 1996 e 1997. O adubo fosfatado foi distribuído em sulcos de $15 \mathrm{~cm}$ de profundidade, abertos lateralmente às linhas de plantio no sentido da projeção da copa no primeiro ano, e a lanço, afastado um metro da linha de seringueira, e dirigido ao retângulo correspondente à área útil nos demais anos. Como fontes de $\mathrm{N}$ e $\mathrm{K}$, foram utilizados, respectivamente, o sulfato de amônio e o cloreto de potássio, sendo os mesmos misturados e aplicados a lanço no retângulo correspondente à área útil de cada planta, sendo a metade aplicada no mesmo período da adubação fosfatada e a outra, quatro meses após.

Nas subparcelas, foram utilizados dois sistemas de explotação da seringueira, sendo os mesmos representados pelas seguintes notações: (S1) ${ }^{1 / S} \mathrm{~d} / 46$ d/7 ET 2,5\% LaPa 1/1 10/y e (S2) 1/S d/6 6 d/7 ET $5,0 \% \mathrm{LaPa} 1 / 1$ 10/y. A explotação foi conduzida no painel $\mathrm{BO}-2$, à altura de $0,90 \mathrm{~m}$ do solo, e a sangria procedida de acordo com as normas preconizadas por Virgens Filho e Castro (1986). Em todos os tratamentos, as plantas foram "sangradas" com corte em meio espiral do tronco ( $(\boldsymbol{B})$, sendo aquelas da subparcela d/4 $6 \mathrm{~d} / 7$, a cada quatro, quatro e cinco dias, respectivamente, e da $\mathrm{d} / 66 \mathrm{~d} / 7$, a cada sete dias. As estimulações foram de dez por ano (10/y) em ambos os sistemas. O estimulante "Ethephon" foi aplicado na região compreendida entre o canal de sangria sobre o cernambi e a casca em regeneração, logo acima desse ( $\mathrm{LaPa} 1 / 1)$. Anualmente foi feito o balanceamento do painel Aensualgrąate foram realizadas mensurações do crescimento do perímetro do tronco e da espessura da casca a 1,50 m do solo, assim como foi avaliado o comprimento dos cortes secos (CCS), de acordo com as metodologias descritas por Jobe-Duval (1986). No terceiro ano, foram determinadas as características físicas e químicas do látex ( $\mathrm{pH}$, sacarose, tióis, $\mathrm{Mg}, \mathrm{P}$, conteúdo de borracha seca - DRC), conforme os procedimentos preconizados por Jacob et al. (1987).

A utilização da análise por contraste permitiu avaliar o efeito da calagem, da adubação NPK, NP e K, e da interação NP vs. K. Esse mesmo procedimento foi usado para avaliar os sistemas de explotação e suas intera- ções com todos os tratamentos. Foram utilizados análise de variância e teste de Tukey (5\%), para comparação dos contraste entre as médias (GOMES, 1990).

\section{RESULTADOS E DISCUSSÃO}

\section{Perímetro do tronco}

As médias do perímetro do tronco e os incrementos registrados entre o primeiro e segundo ano, e deste para o terceiro ano, são mostrados na Tabela 1. Observa-se que o aumento médio do perímetro do tronco obtido nas duas avaliações foi de 4,0 cm, passando de 59,6 para $63,6 \mathrm{~cm}$. Esse incremento ocorreu preferencialmente devido ao ritmo de explotação implementado pelos sistemas $1 / \mathrm{S} \mathrm{d} / 46$ $\mathrm{d} / 7$ ET e ${ }^{1 / \delta} \mathrm{d} / 66 \mathrm{~d} / 7 \mathrm{ET}$, o que permitiu médias de produção superiores a $1.500 \mathrm{~kg}$ de borracha seca ha ${ }^{-1} \mathrm{ano}^{-1}$, independente do tratamento. Não foi observado efeito de interação adubação versus sistemas de explotação, bem como não houve crescimento diferenciado na comparação entre os dois sistemas (Tabela 2). Verifica-se que o tratamento ' $\mathrm{N}_{1} \mathrm{P}_{1} \mathrm{~K}_{2}+$ calagem' (anos 1 a 2) e o tratamento ' $\mathrm{N}_{0} \mathrm{P}_{0} \mathrm{~K}_{0}+$ calagem' (anos 2 a 3 e anos 1 a 3 ) tiveram maiores incrementos no perímetro do tronco que os demais (Tabela 1). Reis (1979), estudando o efeito da adubação com nitrogênio, fósforo, potássio e da calagem no crescimento da seringueira até quatro anos, no sul da Bahia, observou que somente a adubação com $\mathrm{P}$ aumentou significativamente o perímetro do tronco, fato esse não observado com nitrogênio, potássio e calagem.

\section{Espessura da casca}

As medidas da espessura da casca e os incrementos registrados são mostrados na Tabela 1 . A variação nas doses de $\mathrm{K}$, na presença $\mathrm{N}_{1} \mathrm{P}_{1}$, mostrou efeito quadrático entre o primeiro e o segundo ano, e linear entre o segundo e terceiro ano (Figura 1). O aumento da espessura da casca com aplicação de calcário e potássio pode ser atribuído, principalmente, à deficiência relativa maior desses elementos no solo. O efeito do cálcio, elemento constituinte dos pectatos que formam a lamela média na parede celular, é, portanto, importante nos sítios onde há atividade meristemática. Segundo Shorrocks (1979), cristais de oxalato de cálcio, drusas, são freqüentemente encontrados na casca da seringueira e são escassos em plantas deficientes nesse elemento. Quanto ao efeito do potássio, há um aumento significativo, tendo em vista que a ausência desse elemento pode limitar a espessura da casca e o número de vasos laticíferos, como reportado por Pushparajah (1969) e Compagnon (1986). 
TABELA 1 - Médias do perímetro do tronco (PT) e da espessura da casca (EC) das seringueiras com os respectivos incrementos (IPT e IEC).

\begin{tabular}{|c|c|c|c|c|c|c|c|c|c|c|c|c|}
\hline \multirow[b]{2}{*}{ Tratamentos } & \multicolumn{3}{|c|}{ PT } & \multicolumn{3}{|c|}{ IPT } & \multicolumn{3}{|c|}{ EC } & \multicolumn{3}{|c|}{ IEC } \\
\hline & $\begin{array}{c}\text { Ano } \\
1\end{array}$ & $\begin{array}{c}\text { Ano } \\
2\end{array}$ & $\begin{array}{c}\text { Ano } \\
\mathbf{3}\end{array}$ & $\begin{array}{l}\text { Anos } \\
1 \text { a } 2\end{array}$ & $\begin{array}{l}\text { Anos } \\
2 \text { a } 3\end{array}$ & $\begin{array}{l}\text { Anos } \\
1 \text { a } 3\end{array}$ & $\begin{array}{c}\text { Ano } \\
1\end{array}$ & $\begin{array}{c}\text { Ano } \\
2\end{array}$ & $\begin{array}{c}\text { Ano } \\
\mathbf{3}\end{array}$ & $\begin{array}{l}\text { Anos } \\
1 \text { a } 2\end{array}$ & $\begin{array}{l}\text { Anos } \\
2 \text { a } 3\end{array}$ & $\begin{array}{l}\text { Anos } \\
1 \text { a } 3\end{array}$ \\
\hline & & & ---- c & & & - & 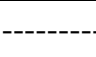 & - & 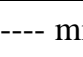 & & & - \\
\hline $\mathrm{N}_{0} \mathrm{P}_{0} \mathrm{~K}_{0}$ & 59,1 & 60,4 & 61,6 & 1,3 & 1,2 & 2,5 & 5,2 & 8,0 & 9,1 & 2,8 & 1,1 & 3,9 \\
\hline $\mathrm{N}_{0} \mathrm{P}_{0} \mathrm{~K}_{0}+\mathrm{Cal}$ & 59,2 & 61,4 & 64,3 & 2,2 & 2,9 & 5,1 & 5,3 & 8,1 & 8,7 & 2,8 & 0,8 & 3,6 \\
\hline $\mathrm{N}_{1} \mathrm{P}_{1} \mathrm{~K}_{0}+\mathrm{Cal}$ & 58,7 & 60,1 & 62,5 & 1,4 & 2,4 & 3,8 & 5,5 & 8,1 & 8,9 & 2,6 & 0,8 & 3,4 \\
\hline $\mathrm{N}_{2} \mathrm{P}_{2} \mathrm{~K}_{0}+\mathrm{Cal}$ & 58,1 & 60,3 & 62,3 & 2,2 & 2,0 & 4,2 & 5,1 & 7,8 & 8,5 & 2,7 & 0,7 & 3,4 \\
\hline $\mathrm{N}_{1} \mathrm{P}_{1} \mathrm{~K}_{1}+\mathrm{Cal}$ & 59,8 & 61,9 & 64,2 & 2,1 & 2,3 & 4,4 & 5,3 & 8,1 & 8,8 & 2,8 & 0,7 & 3,5 \\
\hline $\mathrm{N}_{1} \mathrm{P}_{1} \mathrm{~K}_{2}+\mathrm{Cal}$ & 61,6 & 63,9 & 65,8 & 2,3 & 1,9 & 4,2 & 5,3 & 8,4 & 9,1 & 3,1 & 0,7 & 3,8 \\
\hline $\mathrm{N}_{2} \mathrm{P}_{2} \mathrm{~K}_{1}+\mathrm{Cal}$ & 60,1 & 61,7 & 64,0 & 1,6 & 2,3 & 3,9 & 5,6 & 8,0 & 8,9 & 2,4 & 0,9 & 3,3 \\
\hline $\mathrm{N}_{2} \mathrm{P}_{2} \mathrm{~K}_{2}+\mathrm{Cal}$ & 60,1 & 62,3 & 63,6 & 2,2 & 1,3 & 3,5 & 5,5 & 8,3 & 8,5 & 2,8 & 0,2 & 3,0 \\
\hline $1 / 8 \mathrm{~d} / 46 \mathrm{~d} / 7 \mathrm{ET}$ & 59,3 & 61,2 & 63,2 & 1,9 & 2,0 & 3,9 & 5,3 & 7,9 & 8,9 & 2,6 & 1,0 & 3,6 \\
\hline${ }^{1 / 8} \mathrm{~d} / 66 \mathrm{~d} / 7 \mathrm{ET}$ & 59,9 & 61,7 & 63,9 & 1,8 & 2,2 & 4,0 & 5,4 & 8,2 & 8,8 & 2,8 & 0,6 & 3,4 \\
\hline Médias gerais & 59,6 & 61,5 & 63,6 & 1,9 & 2,0 & 3,9 & 5,3 & 8,1 & 8,8 & 2,7 & 0,7 & 3,4 \\
\hline C.V. $(\%)$ & 9,70 & 9,00 & 10,50 & 53,70 & 55,80 & 54,75 & 7,40 & 8,22 & 6,03 & 36,78 & 55,29 & 46,03 \\
\hline
\end{tabular}

TABELA 2 - Valores do teste F relativos ao incremento do perímetro do tronco (IPT) e espessura da casca (IEC) da seringueira.

\begin{tabular}{lcccc}
\hline \multirow{2}{*}{ Causas da Variação } & \multicolumn{2}{c}{ IPT } & \multicolumn{2}{c}{ IEC } \\
\cline { 2 - 3 } \cline { 5 - 5 } & Anos 1 e 2 & Anos 2 e 3 & Anos 1 e 2 & Anos 2 e 3 \\
\hline Bloco & 0,11 & 1,77 & 2,34 & 0,82 \\
Adubação & 1,13 & 2,44 & 2,32 & 1,97 \\
Com calagem vs. Sem calagem & 0,00 & $8,03^{*}$ & $4,21^{*}$ & 1,18 \\
Com calagem vs. Sem Cal./Adub. & 3,72 & $5,04^{*}$ & $6,92^{* *}$ & 3,03 \\
$\mathrm{~N}_{0} \mathrm{P}_{0} \mathrm{~K}_{0}$ Cal. vs. Cal+NPK & 0,33 & $6,05^{*}$ & 0,01 & 0,12 \\
$\mathrm{~K}_{0}$ vs. $\mathrm{K}_{1}, \mathrm{~K}_{2}$ & 0,39 & 0,81 & 0,87 & $4,80^{*}$ \\
$\mathrm{~N}_{1} \mathrm{P}_{1}$ vs. $\mathrm{N}_{2} \mathrm{P}_{2}\left(\mathrm{~K}_{0}\right)$ & 1,29 & 0,61 & 0,59 & 0,04 \\
$\mathrm{~K}_{1}$ vs. $\mathrm{K}_{2}$ & 1,24 & 0,69 & 3,93 & 1,32 \\
$\mathrm{~N}_{1} \mathrm{P}_{1}$ vs. $\mathrm{N}_{2} \mathrm{P}_{2}\left(\mathrm{~K}_{1}\right)$ & 0,22 & 0,76 & 0,12 & 0,89 \\
$\mathrm{~N}_{1} \mathrm{P}_{1}$ vs. $\mathrm{N}_{2} \mathrm{P}_{2}\left(\mathrm{~K}_{2}\right)$ & 0,72 & 3,10 & 3,77 & 3,59 \\
Resíduo $(\mathrm{A})$ & 0,86 & 3,46 & 0,61 & 1,36 \\
\hline Sistema de explotação & 0,57 & 1,05 & 1,12 & 0,07 \\
Adubação vs. Sist. de explotação & 0,42 & 0,81 & 1,25 & 1,38 \\
Resíduo $(\mathrm{B})$ & 0,47 & 1,74 & 0,86 & 0,03 \\
\hline Médias gerais & 1,99 & 2,00 & 1,60 & 1,00 \\
C.V.(\%) & 53,70 & 55,80 & 36,78 & 55,29 \\
\hline
\end{tabular}

* $\mathrm{e}^{* *}$ valores significativos ao nível de 5 e $1 \%$, respectivamente.

Ciênc. agrotec., Lavras. V.27, n.6, p.1237-1245, nov./dez., 2003 
(a)

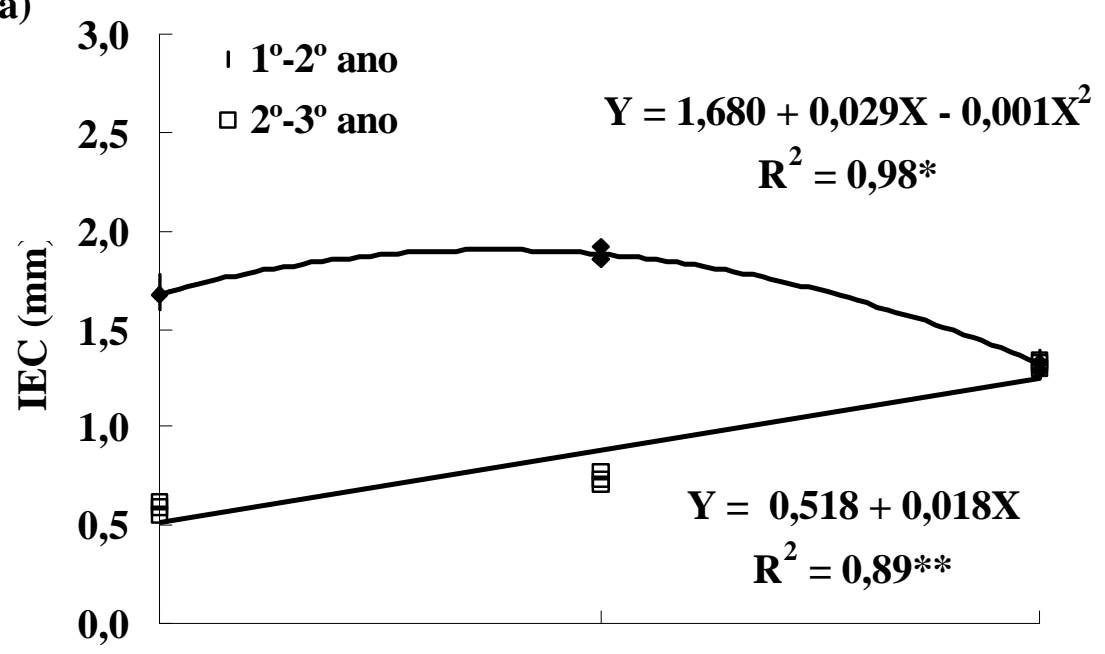

(b) $\mathbf{3 , 0}$

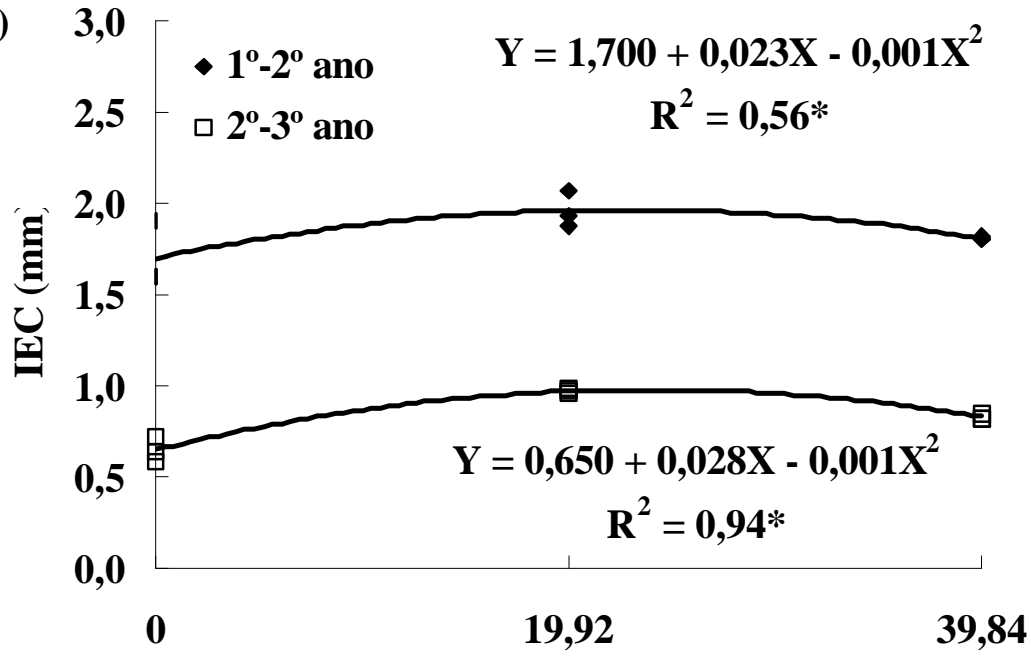

Doses de Potássio (kg ha ${ }^{-1}$ ano $^{-1}$ )

FIGURA 1 - Efeito das doses de potássio sobre o incremento na espessura da casca entre o primeiro e segundo ano e entre o segundo e terceiro ano: (a) Tratamento $\mathrm{N}_{1} \mathrm{P}_{1}$; (b) Tratamento $\mathrm{N}_{2} \mathrm{P}_{2}$. (* e ** significativo a 5 e $1 \%$, respectivamente, pelo teste F).

O incremento na espessura da casca, quando se considera o mesmo clone, tem correlação direta com a expansão da casca macia, local em que se encontram os vasos laticíferos mais jovens, funcionais e produtivos (GOMEZ, 1982). Sob condições de um adequado estado nutricional, pode-se obter um espessamento maior e mais rápido da casca, o que favorece as condições do painel para futuras sangrias. Esse incremento é de grande importância na explotação dos seringais, possibilitando uma melhor distribuição da área de drenagem, o que proporciona maiores produções por planta; também permite uma gestão mais adequada do painel, melhorando a posição do corte na sangria, bem como evita o abaixamento do sangrador até a base do tronco e melhora o rendimento da mão-de-obra.

Ciênc. agrotec., Lavras. V.27, n.6, p.1237-1245, nov./dez., 2003 


\section{Características físicas e químicas do látex}

A concentração de sacarose no látex no tratamento ' $\mathrm{N}_{1} \mathrm{P}_{1} \mathrm{~K}_{2}+$ calagem' foi significativamente superior à obtida com os demais tratamentos (Tabelas 3 e 4). Esse tratamento, além de promover boa resposta na produção, possibilitou uma quantidade de nutrientes necessária ao metabolismo das plantas, o que refletiu em bom suprimento de carboidratos necessários à regeneração do látex. Na comparação entre médias por meio de contrastes, o tratamento ' $\mathrm{N}_{0} \mathrm{P}_{0} \mathrm{~K}_{0}$ ' foi semelhante ao tratamento ' $\mathrm{N}_{0} \mathrm{P}_{0} \mathrm{~K}_{0}+$ calagem', demonstrando que somente a aplicação de calcário não aumenta a concentração dessa variável estudada. O contraste 'calagem vs sem calagem+adubação' mostrou vantagem da 'calagem+adubação' sobre ' $\mathrm{N}_{0} \mathrm{P}_{0} \mathrm{~K}_{0}$ '. No nível $\mathrm{K}_{0}, \mathrm{~N}_{1} \mathrm{P}_{1}$ foi superior a $\mathrm{N}_{2} \mathrm{P}_{2}$, mostrando que há menor demanda de nitrogênio e fósforo na ausência de potássio. Nos níveis $\mathrm{K}_{1}$ e $\mathrm{K}_{2}, \mathrm{~N}_{2} \mathrm{P}_{2}$ superou $\mathrm{N}_{1} \mathrm{P}_{1}$. Não houve diferença entre as doses de potássio (Tabela 3), o que demonstra que a dose $33,2 \mathrm{~kg} \mathrm{ha}^{-1}$ de $\mathrm{K}_{2} \mathrm{O}$ foi suficiente para suprir as necessidades da serin- gueira, resultado observado na maioria das características estudadas (Tabela 3). A concentração de sacarose no látex também foi influenciada pelo efeito da interação entre calagem+adubação e sistemas de

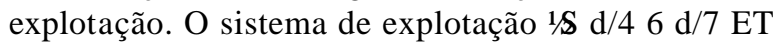
$2,5 \% 10 \mathrm{y}$ apresentou maiores médias de sacarose no látex que o sistema 1/\$ d/6 d d/7 ET 5\% 10y. Com esse resultado, verifica-se que doses adequadas de "Ethephon" compensam a redução da freqüência de "sangria" do segundo sistema, proporcionando maiores produções e, desse modo, aumenta a demanda de sacarose, visando à síntese isoprênica e à produção de borracha seca.

A concentração de fósforo inorgânico na presença de potássio foi influenciada pela adubação fosfatada. Na ausência de $\mathrm{K}$, a dose $\mathrm{N}_{2} \mathrm{P}_{2}$ superou $\mathrm{N}_{1} \mathrm{P}_{1}$, ao passo que, na presença de $\mathrm{K}_{1}$ e $\mathrm{K}_{2}$, não houve diferença significativa (Tabelas 3 e 4). A calagem não promoveu efeito sobre o aumento da concentração de $\mathrm{P}$ no látex. Em contrapartida, houve incremento na concentração de $\mathrm{Mg}$, com média estatisticamente superior à 'testemunha absoluta'.

TABELA 3 - Valores do teste F da análise de variância e de contrastes dos tratamentos relativos as características físicas e químicas do látex.

\begin{tabular}{lcccccccc}
\hline \multicolumn{1}{c}{ Causas de variação } & G.L. & Prod. & pH & Sacarose & P inorg. & Mg & Tióis & DRC $^{\mathbf{1}}$ \\
\hline Adubação & 7 & $5,57^{*}$ & $6,72^{* *}$ & $41,1^{* *}$ & $6,9^{* *}$ & 1,86 & $2,56^{* *}$ & 0,50 \\
Com calagem vs. Sem Cal. & 1 & 0,54 & $31,2^{* *}$ & $1546^{* *}$ & 0,1 & $9,52^{*}$ & 0,14 & 4,02 \\
Com calagem $v$ s. Sem Cal./Adub. & 1 & 1,12 & $43,5^{* *}$ & $7617^{* *}$ & $28,9^{* *}$ & $7,70^{*}$ & 0,01 & 2,36 \\
$\mathrm{~N}_{0} \mathrm{P}_{0} \mathrm{~K}_{0}$ Cal. vs. Cal+NPK & 1 & 1,59 & 0,85 & $1657^{* *}$ & $40,9 * *$ & 2,28 & 0,25 & 1,66 \\
$\mathrm{~K}_{0}$ vs. $\mathrm{K}_{1}, \mathrm{~K}_{2}$ & 1 & 1,53 & 6,07 & $23103^{* *}$ & $14,9^{* *}$ & 0,03 & 0,86 & 0,02 \\
$\mathrm{~N}_{1} \mathrm{P}_{1}$ vs. $\mathrm{N}_{2} \mathrm{P}_{2}\left(\mathrm{~K}_{0}\right)$ & 1 & 0,02 & 3,24 & $58061^{* *}$ & $4,4^{*}$ & 3,60 & 2,31 & 0,35 \\
$\mathrm{~K}_{1}$ vs. $\mathrm{K}_{2}$ & 1 & 1,96 & 2,81 & $64414^{* *}$ & $7,3^{*}$ & $10,3^{* *}$ & $4,61^{*}$ & 0,01 \\
$\mathrm{~N}_{1} \mathrm{P}_{1}$ vs. $\mathrm{N}_{2} \mathrm{P}_{2}\left(\mathrm{~K}_{1}\right)$ & 1 & 0,04 & 3,13 & $4593^{* *}$ & 0,1 & 2,66 & 0,14 & 0,01 \\
$\mathrm{~N}_{1} \mathrm{P}_{1} v s . \mathrm{N}_{2} \mathrm{P}_{2}\left(\mathrm{~K}_{2}\right)$ & 1 & 2,61 & 7,55 & $33267^{* *}$ & 0,6 & 1,23 & $24,3 * *$ & 0,14 \\
Sistema de explotação & 1 & 0,03 & 0,32 & $110,5^{*}$ & $22,1^{*}$ & 0,00 & 2,40 & 1,27 \\
Adub. vs. Sist. De explotação & 7 & 1,19 & 2,06 & $129,3^{*}$ & 1,5 & 0,44 & 1,27 & 0,55 \\
\hline
\end{tabular}

* $\mathrm{e}^{* *}$ valores significativos ao nível de 5 e $1 \%$, respectivamente.

${ }^{1}$ Conteúdo de borracha seca.

Ciênc. agrotec., Lavras. V.27, n.6, p.1237-1245, nov./dez., 2003 
TABELA 4 - Características físicas e químicas do látex, comprimento do corte seco e diagnose em condição de explotação em razão dos tratamentos ${ }^{1}$.

\begin{tabular}{|c|c|c|c|c|c|c|c|c|c|}
\hline Tratamentos & pH & Sacarose & $\begin{array}{c}\mathbf{P} \\
\text { inorg. }\end{array}$ & Tióis & Mg & $\mathbf{M g} / \mathbf{P}$ & DRC $^{2}$ & $\mathrm{CCS}^{3}$ & Condição \\
\hline & & \multicolumn{4}{|c|}{------- mmol L ${ }^{-1}$} & & \multicolumn{2}{|c|}{------ \% ------ } & \\
\hline $\mathrm{N}_{0} \mathrm{P}_{0} \mathrm{~K}_{0}$ & $7,2 \mathrm{a}$ & $11,3 \mathrm{c}$ & $3,92 \mathrm{c}$ & $0,72 \mathrm{ab}$ & $26,2 b$ & 6,6 & $44,0 \mathrm{a}$ & 0,12 & Subexplotação \\
\hline $\mathrm{N}_{0} \mathrm{P}_{0} \mathrm{~K}_{0}+\mathrm{Cal}$ & $6,8 \mathrm{a}$ & $11,8 \mathrm{c}$ & $3,86 \mathrm{c}$ & $0,75 \mathrm{ab}$ & $31,1 \mathrm{a}$ & 8,0 & $51,1 \mathrm{a}$ & 0,03 & Subexplotação \\
\hline $\mathrm{N}_{1} \mathrm{P}_{1} \mathrm{~K}_{0}+\mathrm{Cal}$ & $7,0 \mathrm{a}$ & $13,2 b$ & $4,97 b c$ & $0,70 \mathrm{~b}$ & $30,7 \mathrm{ab}$ & 6,2 & $49,0 \mathrm{a}$ & 0,07 & Subexplotação \\
\hline $\mathrm{N}_{2} \mathrm{P}_{2} \mathrm{~K}_{0}+\mathrm{Cal}$ & $6,8 \mathrm{a}$ & $9,6 \mathrm{e}$ & $6,00 \mathrm{ab}$ & $0,80 \mathrm{ab}$ & $27,7 \mathrm{ab}$ & 4,6 & $47,0 \mathrm{a}$ & 0,06 & Subexplotação \\
\hline $\mathrm{N}_{1} \mathrm{P}_{1} \mathrm{~K}_{1}+\mathrm{Cal}$ & $7,0 \mathrm{a}$ & $13,6 \mathrm{~b}$ & $6,21 \mathrm{ab}$ & $0,65 b$ & $26,3 b$ & 4,2 & $48,0 \mathrm{a}$ & 0,03 & Subexplotação \\
\hline $\mathrm{N}_{1} \mathrm{P}_{1} \mathrm{~K}_{2}+\mathrm{Cal}$ & $6,9 a$ & $14,6 \mathrm{a}$ & $6,15 \mathrm{ab}$ & $0,67 b$ & $28,8 \mathrm{ab}$ & 4,7 & $47,6 \mathrm{a}$ & 0,00 & Subexplotação \\
\hline $\mathrm{N}_{2} \mathrm{P}_{2} \mathrm{~K}_{1}+\mathrm{Cal}$ & $6,7 \mathrm{a}$ & $10,0 \mathrm{e}$ & $7,30 \mathrm{a}$ & $0,60 \mathrm{~b}$ & $30,3 \mathrm{ab}$ & 4,2 & $47,0 \mathrm{a}$ & 0,09 & Subexplotação \\
\hline $\mathrm{N}_{2} \mathrm{P}_{2} \mathrm{~K}_{2}+\mathrm{Cal}$ & $6,9 \mathrm{a}$ & $12,8 \mathrm{~b}$ & $6,93 \mathrm{a}$ & $0,92 \mathrm{a}$ & $32,0 \mathrm{a}$ & 4,6 & $48,2 \mathrm{a}$ & 0,01 & Subexplotação \\
\hline $1 / 2 \mathrm{~S} \mathrm{~d} / 46 \mathrm{~d} / 7$ ET $2,5 \%$ & $6,9 \mathrm{a}$ & $13,2 \mathrm{a}$ & $4,85 b$ & $0,76 \mathrm{a}$ & $29,1 \mathrm{a}$ & 6,0 & $46,7 \mathrm{a}$ & 0,04 & Subexplotação \\
\hline $1 / 8 \mathrm{~d} / 66 \mathrm{~d} / 7$ ET $5 \%$ & $6,9 \mathrm{a}$ & $11,1 \mathrm{~b}$ & $6,48 \mathrm{a}$ & $0,69 \mathrm{a}$ & $29,7 \mathrm{a}$ & 4,6 & $48,8 \mathrm{a}$ & 0,06 & Subexplotação \\
\hline Médias gerais & $6,9 \mathrm{a}$ & 12,1 & 5,61 & 0,72 & 29,1 & 5,4 & 47,7 & 0,05 & \\
\hline DMS & 0,2 & 0,8 & 1,4 & 0,2 & 4,6 & & 7,6 & & \\
\hline C.V. $(\%)$ & 2,74 & 6,37 & 24,50 & 24,30 & 15,83 & & 15,9 & & \\
\hline
\end{tabular}

${ }^{1}$ Médias seguidas por letras distintas na mesma linha diferem a $5 \%$ de probabilidade pelo teste de Tukey. ${ }^{2}$ Conteúdo de borracha seca. ${ }^{3}$ Comprimento de corte seco.

A concentração de magnésio foi maior na dose $\mathrm{K}_{2}$, o que se deve à aplicação de calcário dolomítico $(24 \%$ de $\mathrm{CaO}$ e $16 \%$ de $\mathrm{MgO}$ ), que aumentou a disponibilidade desse elemento no solo e manteve o teor alto nas folhas (3,3 $\mathrm{g}$ $\mathrm{kg}^{-1}$ ). A relação $\mathrm{Mg} / \mathrm{P}$ foi maior nos tratamentos 'testemunha absoluta', ' $\mathrm{N}_{0} \mathrm{P}_{0} \mathrm{~K}_{0}+$ calagem' e ' $\mathrm{N}_{1} \mathrm{P}_{1} \mathrm{~K}_{1}+$ calagem', apresentando valores 6,6, 8,1 e 6,2, respectivamente. Esses valores são mais elevados que o considerado adequado para manter a estabilidade do látex, que é de 5,4 (SHORROCKS, 1979). Nos demais tratamentos, verificam-se valores entre 4,2 e 4,7 (Tabela 4).

Os maiores valores de $\mathrm{pH}$ do látex foram registrados nos tratamentos 'testemunha absoluta' e ' $\mathrm{N}_{2} \mathrm{P}_{2} \mathrm{~K}_{1}+$ calagem'. A calagem e/ou adubação proporcionaram um $\mathrm{pH}$ mais baixo em relação à 'testemunha absoluta'. Na presença de $\mathrm{N}_{2} \mathrm{P}_{2}$, a dose $\mathrm{K}_{1}$ superou a $\mathrm{K}_{2}$ (Tabelas 3 e 4).

O conteúdo de borracha seca (DRC) do látex não apresentou diferença significativa por efeito dos trata- mentos com adubação e calagem (Tabelas 3 e 4). Os valores médios bastante elevados para DRC foram devidos ao método utilizado, uma vez que essa variável foi um dos últimos a serem determinados, havendo defasagem entre a coleta no campo, a pesagem em laboratório e a secagem na estufa.

A concentração de tióis no látex foi aumentada com o incremento das doses de NPK (Tabelas 3 e 4), ao passo que o maior comprimento de corte seco (CCS), em porcentagem, foi obtido na 'testemunha absoluta' (Tabela 4). Pelos resultados relativos ao efeito da adubação e calagem nas características fisico-químicas do látex, verifica-se que o potássio é o elemento "chave" na nutrição da seringueira, o que é explicado pela sua função metabólica nos processos ligados à translocação de compostos fotossintetizados pelo floema, à síntese isoprênica e à duração do fluxo de látex após a sangria (ESCHBACH e LACROTTE, 1989; JACOB et al., 1989). A calagem e adubação fosfatada aumentaram a 
concentração de $\mathrm{Mg}$ e $\mathrm{P}$ no látex, respectivamente, e proporcionaram uma melhor relação $\mathrm{Mg} / \mathrm{P}$ do que a calagem isolada (Tabela 4). Segundo Marschner (1995), o magnésio ativa quase todas as enzimas fosforilativas que formam pontes entre o ATP ou ADP com a molécula da enzima. As transferências de energia desses dois compostos são fundamentais nos processos de fotossíntese, reações de síntese de compostos orgânicos e absorção iônica.

Avaliação das condições de explotação da seringueira

A condição de explotação foi definida em função da análise das médias observadas para as características fisiológicas, que são apresentadas na Tabela 4. Essa metodologia teve suporte em informações descritas por Jacob et al. (1989), cujo modelo foi proposto para o clone GT 1.

Existe uma condição de subexplotação das plantas em todos os tratamentos, indicada, sobretudo, pela boa disponibilidade de sacarose, valores elevados de $\mathrm{pH}$ $(7,05)$, e DRC, valores de fósforo inorgânico em torno de 4,0 a 6,0 mmol L $\mathrm{m}^{-1}$, salvo na dose $35 \mathrm{~kg} \mathrm{ha}^{-1}$ ano $^{-1}$ de $\mathrm{P}_{2} \mathrm{O}_{5}$, que ficou na faixa de $7,0 \mathrm{mmol} \mathrm{L} \mathrm{L}^{-1}$, uma relação $\mathrm{Mg} / \mathrm{P}$ maior que 1 e valores baixos de cortes secos (CCS). Na comparação entre sistemas de explotação, as diferenças não são marcantes, sugerindo uma condição de subexplotação em ambos os sistemas.

\section{CONCLUSÕES}

a) $\mathrm{O}$ incremento no perímetro do tronco não é influenciado pelo efeito de interação entre 'adubação+calagem' vs. 'sistemas de explotação'.

b) $\mathrm{O}$ aumento da espessura da casca é afetada pelos tratamentos ' $\mathrm{N}_{0} \mathrm{P}_{0} \mathrm{~K}_{0}+$ calagem' e 'calagem+adubação', promovendo incrementos significativos em relação à testemunha absoluta $\left(\mathrm{N}_{0} \mathrm{P}_{0} \mathrm{~K}_{0}\right.$ sem calagem).

c) Plantas submetidas ao tratamento $\mathrm{N}_{1} \mathrm{P}_{1} \mathrm{~K}_{0}+$ calagem e ao sistema ${ }^{1 / \delta} \mathrm{d} / 66 \mathrm{~d} / 7$ ET 5\% 10/y apresentam maiores concentrações de sacarose no látex.

d) A aplicação de calcário, juntamente com adubação de NPK, não aumentou o DRC.

\section{REFERÊNCIAS BIBLIOGRÁFICAS}

CARDOSO, M. Seringueira. In: RAIJ, B.; SILVA, N. M.; BATAGLIA, O. C.; QUAGGIO, J. A.; HIROCE, R.; CANTARELLA, H.; BELLINAZZI JUNIOR, R.;
DECHEN, A. R.; TRANI, P. E. (Eds.). Recomendação de adubação e calagem para o Estado de São Paulo. Campinas: Instituto Agronômico de Campinas, 1992. p. 85. (Boletim Técnico, 100).

COMPAGNON, P. Fertilisation. In: COMPAGNON, P. (Ed.). Le caoutchouc naturel. Paris: Maisonneuve et Larose, 1986. p. 297-314.

ESCHBACH, J. M.; LACROTTE, R. Factors influencing response to hormonal yield stimulation: limits of this stimulation. In: D'AUZAC, J.; JACOB, J. L.; CHRESTIN, H. (Eds.). Physiology of rubber tree latex. Boca Raton: CRC, 1989. p. 321-330.

FERRAND, M. Phytotechne de l'Hevea brasiliensis. Paris: Jules Duculot, 1944. 435 p.

FURTADO, E. L.; SILVEIRA, A. P. Doenças do painel de sangria da seringueira. In: BERNARDES, M. S. (Ed.). Sangria da seringueira. Piracicaba: FEALQ, 1990. p. 111-125.

GOMES, F. P. Curso de estatística experimental. Piracicaba: Nobel, 1990. 468 p.

GOMEZ, J. Anatomy of Hevea and it's influence on latex production. Kuala Lumpur: Malaysia Rubber Research on Development Board, 1982. 76 p.

JACOB, J. L.; LACROTTE, R.; SERRES, E.; ROUSSEL, D. Le parametres physiologiques du latex d'Hevea brasiliensis. Paris: IRCA, 1987. 169 p.

JACOB， J. L.; PRÉVÔT, J. C.; ROUSSEL, D.; LACROTTE, R.; ESCHBACH, J. M. Le diagnostic du latex. Plantations, recherché, développment, Paris, v. 2, p. 33-37, 1995.

JACOB, J. L.; PRÉVÔT, J. C.; ROUSSEL, D.; LACROTTE, R.; SERRES, E.; D'AUZAC, J.; ESCHBACH, J. M.; OMONT, H. Yield limiting factors, latex physiological parameters, latex diagnosis, and clonal typology. In: D'AUZAC, J.; JACOB, J. L.; CHRESTIN, H. (Eds.). Physiology of rubber tree latex. Boca Raton: CRC, 1989. p. 345-382.

JACOB， J. L.; SERRES, E.; PRÉVÔT, J. C.; LACROTTE, R.; VIDAL, A.; ESCHABACH, J. M.; D'AUZAC, J. Mise au point du diagnostic latex chez l'Hevea. Agritop, [S.1.], v. 12, p. 97-115, 1988. 
JOBE-DUVAL, B. J. Contribuition à l'etude des phénomènes d'encoche sèche d'Hevea brasiliensis Muell. Arg. Epidemiologie, facteurs de risque et méthodes de lutte. Paris: IRCA, 1986. 62 p.

KEKWICK, R. G. O. The formation of polyisoprenoids in Hevea latex. In: D'AUZAC, J.; JACOB, J. L.; CHRESTIN, H. (Eds.). Physiology of rubber tree latex. Boca Raton: CRC, 1989. p. 145-164.

MAINSTONE, B. J. Manuring of Hevea. VI: some long term manuring effects, with special reference to phosphorus, in one of the DUNLOP (Malaysia) experiments. Empire Journal of Experimental Agriculture, Oxford, v. 31, p. 175-185, 1963.

MARSCHNER, H. Mineral nutrition of highers plants. London: Academic, 1995. 888 p.

NASCIMENTO, C. M.; PEREIRA, M. A. M. G. Atlas climatológico do Estado de São Paulo: 1977-1986. Campinas: Fundação Cargill, 1988. 93 p.

ORTOLANI, A. A.; PEDRO JUNIOR, M. J.; ALFONSI, R. R.; CAMARGO, N. B. D.; BRUNINI, O. Aptidão agroclimática para a regionalização da heveicultura no Brasil. In: SEMINÁRIO BRASILEIRO SOBRE RECOMENDAÇÕES DE CLONES DE SERINGUEIRA, 1., 1982, Brasília. Anais... Brasília: EMBRAPA, 1983. p. 17-28.

PUSHPARAJAH, E. Response in growth and yield of Hevea brasiliensis to fertilizer application on Rengan series soils. Journal of the Rubber Research Institute of Malaya, Kuala Lumpur, v. 21, p. 165-172, 1969.

REIS, E. L. Influência da aplicação de nitrogênio, fósforo e potássio sobre o desenvolvimento da seringueira (Hevea brasiliensis Muell. Arg) no sul da Bahia. 1979. 61 f. Dissertação (Mestrado) - Escola Superior de Agricultura Luiz de Queiroz, Universidade de São Paulo, Piracicaba, 1979.

REIS, E. L.; CABALA ROSAND, F. P. Eficiência dos fertilizantes aplicados na fase de pré e pós sangria da seringueira. Revista Theobroma, Itabuna, v. 18, n. 3, p. 189-200, 1988.

SETZER, J. Contribuição para o estudo do clima do Estado de São Paulo. São Paulo: Escolas Profissionais Salesianas, 1946. 239 p.

SHORROCKS, V. M. Deficiências minerais em Hevea e em plantas de cobertura associadas. Brasília: SUDHEVEA, 1979. 76 p.

VIRGENS FILHO, A. C.; CASTRO, P. R. C. Sangria da seringueira (Hevea spp.). In: SIMPÓSIO SOBRE A CULTURA DA SERINGUEIRA, 1., 1986, Piracicaba. Anais... Campinas: Fundação Cargill, 1986. p. 271315 .

WATSON, G. A. Nutrition. In: WEBSTER, C. C.; BAULKWILL, W. J. (Eds.). Hevea. New York: Longman, 1989. p. 125-163. 